\title{
Investigating The Lived Experience of People With Diabetic Foot Ulcers Receiving Podiatry Care in a Tertiary Hospital Clinic
}

Emilee Kim Ming Ong ( $\sim$ ongey008@mymail.unisa.edu.au )

University of South Australia https://orcid.org/0000-0001-7691-1263

\section{Caroline Fryer}

University of South Australia

Kristin Graham

University of South Australia

Ryan Scott Causby

University of South Australia

\section{Research Article}

Keywords: Diabetic foot ulcers, Diabetes, Patient experience, Lived experience, Interpretive phenomenological analysis

Posted Date: November 12th, 2021

DOI: https://doi.org/10.21203/rs.3.rs-1055227/v1

License: (9) This work is licensed under a Creative Commons Attribution 4.0 International License. Read Full License 


\section{Abstract \\ Background:}

Diabetic foot ulcers can have physical, social, emotional, and financial impacts on the daily life and wellbeing of many people living with diabetes. Effective treatment of diabetic foot ulcers requires a multifaceted, multi-disciplinary approach involving a podiatrist, other healthcare professionals, and the person with diabetes however, limited research has been conducted on the lived experience of managing a diabetic foot ulcer with podiatry treatment. Therefore, this study aimed to explore the lived experience of receiving podiatric treatment for diabetic foot ulcers in a tertiary care outpatient setting.

\section{Methods:}

Participants with diabetic foot ulcers were purposively recruited from the outpatient podiatry clinic at a tertiary hospital in a metropolitan region of South Australia. Semi-structured interviews were conducted to gain insight into the lived experience of people receiving podiatric treatment for their foot ulcer. Data were analysed using Interpretative Phenomenological Analysis method.

\section{Results:}

Ten participants were interviewed. All were male, with mean age of 69 (SD 15) years and currently undergoing podiatric treatment for a diabetic foot ulcer in a tertiary care setting. Four themes were identified that provide an understanding of the lived experience of participants: 'Trusting the podiatrists with the right expertise', 'Personalised information', 'Happy with the service, not the equipment', and 'It's a long journey'. Participants perceived professional behaviour and demonstrated expertise as key to trusting a podiatrist's care. Information tailored to individual needs was helpful for participants. Offloading devices and ulcer dressings were often burdensome. Podiatric treatment for foot ulcers was a lengthy and onerous experience for all participants.

\section{Conclusions:}

The findings of this study suggest podiatrists can use practical strategies of maintaining consistency in who provides podiatry care for the person, demonstration of high organisational and hygiene standards, and using innovation to adapt information and equipment to suit an individual's lifestyle to support positive experiences of podiatric ulcer care. There is a need for further research to understand how a person's experience of podiatric ulcer treatment differs between genders and healthcare settings to facilitate positive care experiences and reduce treatment burden for all people with diabetes requiring podiatric ulcer treatment. 


\section{Background}

Diabetic foot ulcers (DFUs) have significant physical, social, economic, and psychological impacts for many people living with diabetes. The Diabetic Foot Australia guide (8) estimates that diabetes-related foot disease costs the Australian healthcare system $\$ 4.3$ million daily. A DFU is a full thickness wound in the epithelium that extends into or through the dermis layer and may cause damage to underlying structures including nerves and tissues distal to the ankles $(3,4)$. Twenty-five percent of people living with diabetes will develop a DFU within their lifetime (9). Male gender and neuropathy are key predictors for the development of DFUs (10). The high prevalence of DFUs presents a significant burden on individuals, communities, and healthcare systems around the world.

Poorly managed DFUs can compromise the viability of an entire lower limb. Infection, gangrene, and lower limb amputations are potential complications, with over half of all ulcer cases becoming infected (11). Diabetic ulcers are a common precursor for amputation, with $85 \%$ of lower limb amputations preceded by foot ulceration (12), and a five-year mortality rate of $40 \%$ following a lower limb amputation (13). The poor prognosis for DFUs emphasises the importance for health professionals to have a thorough understanding of the strategies to successfully manage DFUs.

Long term management of a chronic disease such as diabetes mellitus can have widespread effects on individuals, including a negative outlook on life and feelings of sadness, worry, and despair (14). Diabetes introduces a burden on the physical, psychological, and social contributors of quality of life in people living with the disease (15). To the best of our knowledge, current literature investigating how people experience podiatric ulcer treatment within chronic diabetes management is limited.

Effective treatment of a DFU requires a multi-faceted, multi-disciplinary approach involving healthcare professionals and the person with diabetes (2). Management of DFUs involves prevention of infection, wound care, and metabolic control (16). Offloading, in which load is redistributed from high pressure regions on the foot using non-removable casting or removable cast walkers are an important aspect of ulcer treatment (16). Podiatrists play a fundamental role in wound management and patient education focusing on foot selfcare habits for ulcer prevention. However, how this information is received by people with DFUs and how podiatry care is experienced during DFU management remains unclear.

Poor treatment compliance is a common challenge for podiatrists and other health professionals (17). Previous studies including a cross-sectional analysis of 1743 patients with HIV suggested that patientcentred care incorporating patient-practitioner rapport can improve treatment compliance for various health conditions $(18,19)$. However, few studies have investigated how interactions with a podiatrist impact a person's experiences of podiatric treatment.

Multi-disciplinary treatment and management of DFUs can have a broad impact on the physical, social, and emotional wellbeing of the individual (Figure 1). Limitations in lifestyle activities that provided meaning for the person such as leisure or activities of daily living due to ulcer management are well reported in qualitative studies to have negative psychological effects $(1,2,20)$. Yet how this relates to the 
podiatric treatment which people receive for DFUs remains unclear. Offloading management and time commitments to medical appointments for ulcer treatment present significant restrictions in employment for many patients (21). A study using focus group interviews of patients attending a diabetic foot clinic for DFU treatment found that ulcer management introduced financial hardships and loss of stimulation previously provided from work (22). Travel to medical appointments, parking fees, and costs of prescription devices are reported to contribute to the economic burden associated with DFU treatment (2, 23). Foster and Lauver (24) conducted semi-structured interviews of 15 people with DFUs and identified financial burden and future uncertainty as predominant themes in their lives. Despite this, few studies describe the role of podiatric ulcer treatment within this reported burden which limits the capacity of podiatrists to improve care experience for this group of people. This highlights a gap in the current body of professional knowledge. Interpretative Phenomenological Analysis (IPA) is a useful approach to better understand a person's lived experience.

Interpretative phenomenology provides an understanding of the lived experience of a phenomena within a social and temporal context (25). Semi-structured interviews (SSIs) in IPA encourage participants to share experiences of the phenomena which are important to them (5), enabling researchers to collect meaningful data. A thorough understanding of the healthcare experience is essential to develop the most effective patient-centred treatment plan (22) and so IPA research can inform health professional practice. The IPA research method has been used to understand the lived experience of foot-related conditions including posterior tibial tendon dysfunction (25) and plantar heel pain (26). However, no research has been conducted to understand the experience of podiatric ulcer management using this method.

Therefore, this study informed by IPA, aimed to explore the lived experience of people receiving podiatric treatment for diabetic foot ulcers in a tertiary care outpatient setting.

\section{Methods}

This study is reported in accordance with the Consolidated Criteria for Reporting Qualitative Research (COREQ) checklist (27).

\section{Ethical approval}

was obtained from the Central Adelaide Local Health Network (CALHN) and University of South Australia's Human Research Ethics Committee (CALHN reference number 13567) (Appendix 1).

\section{Research design}

A qualitative IPA design was used to collect, analyse, and report the lived experience of people receiving podiatric ulcer treatment. Interpretative phenomenology takes a phenomenological approach to explore the phenomenon of receiving podiatric ulcer care from the perspective of those people who experience it but aims to move beyond description of the experience to an interpretation of its meaning (28). 


\section{Recruitment}

Participants were purposively recruited from an outpatient podiatry clinic at a tertiary care hospital in the metropolitan region of South Australia to ensure they had experience of receiving podiatric ulcer treatment in a tertiary care setting. Ten participants were recruited to provide sufficient data to develop an understanding of meaningful themes (29). Interpretative phenomenological analysis focuses on a small homogenous sample to enable a detailed analysis of each participant's experience (5). Participants who met the inclusion criteria (Table 2) were identified by podiatrists working at the clinic. No relationship was established between the participant and the principal researcher (EO) prior to the interviews.

Table 2

Inclusion/ exclusion criteria for participant selection

\begin{tabular}{|ll|}
\hline Inclusion criteria & Exclusion criteria \\
$\cdot \begin{array}{l}\text {-18+ years old } \\
\text { - Currently has a DFU }\end{array}$ & $\begin{array}{l}\text { - Currently has an ulcer/ history of ulcers of other } \\
\text { non-diabetic disease) }\end{array}$ \\
$\begin{array}{l}\text { - Is receiving treatment for a DFU in a } \\
\text { podiatry department in a tertiary care } \\
\text { health service (hospital) }\end{array}$ & $\begin{array}{l}\text { - Cognitive or communication disorder impairing ability } \\
\text { to describe experience }\end{array}$ \\
& $\begin{array}{l}\text { - Limited English proficiency impairing ability to } \\
\text { describe experience in English }\end{array}$ \\
\hline
\end{tabular}

\section{Data Collection}

An interview time was negotiated after participants provided written and informed consent (Appendix 2). Face-to-face interviews were conducted with the principal researcher (EO) in a meeting room at the clinic and each participant attended one interview. The principal researcher is a podiatry Honours student and was guided by three research supervisors with clinical and research experience in podiatry and physiotherapy. The principal researcher underwent interview training with the research team prior to data collection. A semi-structured interview guide was developed in conjunction with the research team, informed by the study topic, and similar studies using IPA to understand the person's lived experience of podiatric treatment (Appendix 3). Primary open-ended questions and prompts to encourage rich descriptions of the lived experience were included. The semi-structured nature of the interviews allowed for new questions to be developed in response to participants' stories. Demographic data was collected during the interviews. Interviews were audio recorded using a digital voice recorder. Interviews lasted between 10 and 26 minutes (mean 18 minutes). The principal researcher noted each participant's observations and thoughts about their experience in a reflective journal to contribute to interpretation during data analysis. Interviews were transcribed using Descript software (Descript, San Francisco, CA, USA) and checked against the audio recording. 


\section{Data analysis}

Stepwise replication was utilised for dependability (29). Initially, one transcript was chosen to be read separately by the principal researcher (EO) and all three researchers to immerse themselves in the data of a single participant. Initial comments and observations (codes) relating to the research question were identified by the researchers independently (5). The primary researcher then met to discuss the first participant analysis with the other researchers. In these discussions, codes presenting similar ideas were grouped and a theme to describe each group was identified (30). Themes for the single participant were discussed between researchers until agreed upon then summaries describing the salient points of the participant experience were written for each theme (30). Each participant transcript was then analysed without comparison to the themes from other participants' interviews to support focussed interpretation of each lived experience (5). At least two researchers analysed each transcript. When the 10 transcripts had been analysed, themes from all transcripts were compared and discussed by the researchers. Each theme was written on a Post-it ${ }^{\mathrm{TM}}$ note and visually displayed for consideration. The researchers collaboratively identified themes that shared a similar meaning and could be grouped together (30). Four final themes were established by the researchers to provide an understanding of the lived experience data as vividly as possible (Table 3) (7). A summary of the coding tree is displayed in Appendix 4. Direct quotations from the participants were incorporated in the reporting of results to represent the participant voice (5). All coding was recorded in QSR International's NVivo 12 software (released in March 2020) to support mapping of coding and themes.

Table 3

Example of quotes, sub-codes, codes, and themes

\begin{tabular}{|c|c|c|c|}
\hline Quote & Sub-Code & Code & Theme \\
\hline $\begin{array}{l}\text { "Listen to your podiatrist. Do what they tell you" } \\
\text { (P04) }\end{array}$ & $\begin{array}{l}\text { Trusting } \\
\text { expertise }\end{array}$ & $\begin{array}{l}\text { Trusting the } \\
\text { podiatrist's } \\
\text { expertise }\end{array}$ & $\begin{array}{l}\text { Trusting the } \\
\text { podiatrists } \\
\text { with the right } \\
\text { expertise }\end{array}$ \\
\hline $\begin{array}{l}\text { "I surmised that the podiatrist cut too close } \\
\text { because it has happened twice, but l've not been } \\
\text { told what has caused it" (P01) }\end{array}$ & $\begin{array}{l}\text { Podiatrists } \\
\text { aren't trained to } \\
\text { give } \\
\text { information }\end{array}$ & $\begin{array}{l}\text { They don't } \\
\text { tell me what } \\
\text { I want to } \\
\text { know }\end{array}$ & $\begin{array}{l}\text { Personalised } \\
\text { information }\end{array}$ \\
\hline $\begin{array}{l}\text { "They seem to treat everything alright. Their } \\
\text { manner, their interest, everything. Everything } \\
\text { about it is just good" (P05) }\end{array}$ & $\begin{array}{l}\text { Satisfied with } \\
\text { experience }\end{array}$ & $\begin{array}{l}\text { Satisfied } \\
\text { with } \\
\text { podiatry } \\
\text { service and } \\
\text { trust }\end{array}$ & $\begin{array}{l}\text { Happy with the } \\
\text { service, not the } \\
\text { equipment }\end{array}$ \\
\hline $\begin{array}{l}\text { "It's holding me back, but most of all it's holding } \\
\text { the hospital back. I've got to keep on coming } \\
\text { here to get the damage repaired" (P10) }\end{array}$ & $\begin{array}{l}\text { Not a } \\
\text { straightforward } \\
\text { path }\end{array}$ & $\begin{array}{l}\text { Podiatry } \\
\text { care is a } \\
\text { long journey }\end{array}$ & $\begin{array}{l}\text { It's a long } \\
\text { journey }\end{array}$ \\
\hline
\end{tabular}


Credibility, transferability, dependability, and confirmability are strategies to achieve rigour in qualitative studies (31). To maintain credibility, peer debriefing was conducted with the supervisory team throughout data collection to minimise researcher bias (29). A reflexivity debrief session with the research team was conducted prior to data collection to reflect on each researcher's experiences and beliefs about the lived experience of podiatric ulcer care (5). Demographic details of participants and quotations of the patient experience have been provided to allow the reader to determine the transferability of study findings to their local DFU patient population (32). Confirmability was established using an audit trail to clarify the data collection and analysis procedures (29). Generalisability of results is not relevant in IPA as participants are purposively recruited for their individual experiences rather than to represent the experiences of the general DFU patient population (5). Data saturation was not required as IPA aims to collect rich descriptions of individuals' stories to gain an understanding of their lived experience (33). These techniques aimed to privilege the participant voice within their podiatric ulcer treatment to ensure the findings accurately reflect each participant's lived experience (5).

\section{Results}

Ten participants were recruited during December 2020. Demographic data of the participants are listed in Table 4 and Appendix 5. All participants were male, with a mean age of 69.4 years and standard deviation of 15.3 years. Seventy percent of participants were retired and 30\% were unemployed. Seventy percent were living at home with a partner or family member. Eighty percent of participants had been receiving podiatric ulcer management for over nine months, with $70 \%$ of participants reporting a history of ulcer infection. Four themes were identified from data analysis and are displayed in Figure 2. 
Table 4

Participant demographic data

\begin{tabular}{|lllllll|}
\hline $\begin{array}{l}\text { Participant } \\
\text { Code }\end{array}$ & Age & $\begin{array}{l}\text { Employment } \\
\text { Status }\end{array}$ & $\begin{array}{l}\text { Living } \\
\text { Arrangements }\end{array}$ & $\begin{array}{l}\text { Duration of } \\
\text { Ulcer } \\
\text { (months) }\end{array}$ & $\begin{array}{l}\text { Duration of Ulcer } \\
\text { Treatment (months) }\end{array}$ & $\begin{array}{c}\text { Prior } \\
\text { Ulcer }\end{array}$ \\
\hline P01 & 76 & Retired & With a partner & $3-4$ & $2-3$ & Yes, \\
\hline P02 & 95 & Retired & With a partner & $9+$ & $9+$ & Yes, \\
\hline P03 & 49 & Unemployed & Alone & $8-9$ & $9+$ & Yes, \\
\hline P04 & 59 & Retired & With a partner & $3-4$ & $3-4$ & No \\
\hline P05 & 74 & Retired & With a carer & $9+$ & $9+$ & No \\
\hline P06 & 84 & Retired & Alone & $9+$ & $9+$ & No \\
\hline P07 & 63 & Retired & With a partner & $9+$ & $9+$ & Yes, \\
\hline P08 & 51 & Unemployed & With family & $9+$ & $9+$ & Yes, \\
\hline P09 & 83 & Retired & With a partner & $9+$ & $9+$ & No \\
\hline P10 & 60 & Unemployed & With family & $9+$ & $9+$ & \\
\hline
\end{tabular}

\section{Theme 1. Trusting the podiatrists with the right expertise}

Participants described the importance of trusting the podiatrist involved in their ulcer management. This trust was earnt through the person perceiving the podiatrist to demonstrate professionalism in communication and expertise in ulcer debridement and dressings. Participants trusted the footcare advice of podiatrists when a strong rapport was established. This trust helped participants to be confident in following the podiatrist's instructions.

"They're the experts, so take their advice as much as possible and just make sure you do the right things" (P03).

"They know what they're doing, they know more than me. I don't know what they are doing anyways" (P02).

Participants felt respected during their ulcer treatment when podiatrists provided treatment individualised to their needs.

"People treated me how I felt I'd like to be treated" (P06). 
In their expectations of expertise, participants lost trust when a podiatrist was perceived to not demonstrate the knowledge and skills expected of an expert. This included unsatisfactory debridement technique or poor infection control, which participants believed had consequences for their ulcer healing and risk of developing future ulcers. Several participants reported their trust for podiatry tested by negative care experiences. Podiatrists "cutting too deep" was believed to be the cause of ulceration for three participants who felt conflicted by this loss of trust in a professional providing ulcer care. One participant experienced a setback when a change of dressing by a different podiatrist caused an infection in the ulcer.

"If I hadn't gone to the podiatrist in the first place, I might never have had an ulcer" (P01).

Participants felt that the expertise of the treating podiatrist and the use of the correct ulcer dressings were vital for positive treatment outcomes.

"I've been very happy with the way he's treated it with that little red sheet. They just stick that on and then put on a dressing. That seems to be working" (P06).

Consistency associated with being treated by the same trusted podiatrist assured participants that the podiatrist was familiar with their ulcer and had the knowledge to provide the most effective ulcer treatment for them.

\section{"It makes a big difference to who you know, and how you get on with them" (P06).}

Many participants were hesitant to change podiatrists as they experienced distrust and concerns about possible negative repercussions of these changes on their ulcer. Yet, this consistency was not perceived to be within their control in the tertiary health setting.

"Every time I get a good one, they get transferred" (P02).

Different experiences between community podiatry clinics and hospital podiatry services were emphasised. Participants felt more trusting and comfortable with the podiatry ulcer care provided in the hospital and associated this with the professionalism and knowledge demonstrated by the hospital podiatrists. Three participants were particularly appreciative of the innovation shown by podiatrists in the hospital in developing personalised treatment plans to suit their goals and minimise lifestyle interruptions.

"She (podiatrist) is trying everything. If it doesn't work, it's certainly not her fault or the hospital's fault" (P07).

Several participants reported their trust for community podiatry services being tested due to negative experiences involving poor infection control and lack of podiatrist-patient rapport. These participants noted a difference in quality of care provided between the hospital and community podiatry services which dissuaded them from using community services. 
"They discharged me and sent me up the road, where I see a separate podiatrist. Like an outsource podiatrist. But then obviously the problem came back" (P03).

\section{Theme 2. Personalised information}

Information provided by podiatrists needed to be meaningful to the person's individual concerns, beliefs, and lifestyle to be perceived as helpful.

"The first thing I would say to them, tell the person how they got the ulcer" (P01).

Six participants had a strong understanding of why they had an ulcer. Some associated problems with their blood supply with ulcer development. Risks of amputation were acknowledged as potential consequences of a poorly managed DFU.

"You know with diabetics, it's not one problem. It's eyes, feet, ulcers, blood pressure. The heart as well" (P03).

In contrast, knowledge about ulcer aetiology and management was not a strong component of the experience of care for four participants.

"I was just stumped. It didn't make sense to me. That something was going on with my feet" (P06).

Information from the podiatrist was recognised as important to prevent ulcer recurrence and amputations, however, not all participants believed they had received the relevant information to do so.

"The people, they communicate, but they're not telling me what I want to know. I'm not here to grill them" (P01).

An absence of personalised information in a participant's experience created uncertainty for them about the value of podiatric ulcer treatment and their treatment outcomes.

\section{Theme 3. Happy with the service, not the equipment}

Overall, participants were satisfied with the delivery of podiatric treatment they had received in the hospital clinic for their ulcer but were burdened by the equipment prescribed and self-management expected by podiatrists between appointments. Podiatric ulcer treatment was generally perceived as routine, comfortable, and uneventful.

"It's fairly stress-free. It was easy to get in and out. Not too much drama" (P04).

Social interaction with the podiatrist during ulcer treatment was a highlight of the podiatric experience for some participants.

"It's an outing, I'm usually home anyway. I go and have a coffee with some friends there every morning just about, then I'm home for the rest of the day" (P02). 
In contrast to the service experience, the prescription of an offloading footwear device was perceived to be burdensome. Most participants who had been prescribed this equipment displayed an understanding of its rationale for offloading to allow the ulcer to heal and their responsibilities in managing the equipment between podiatry appointments. Yet they found compliance with the equipment to be a significant challenge and inconvenience. Despite this burden most acknowledged the equipment benefitted their ulcer healing and they were attempting to use the equipment as instructed.

"Can't do things like you normally could because of risk of infections" (P04).

"The secret to success is wearing the moonboot" (P07).

The challenges of compliance with prescribed equipment were perceived to not always be understood by the treating podiatrist. One participant presented a façade of compliance with prescribed socks for appointments when they perceived their frustrations and inconvenience was not accepted.

"They (podiatrist) didn't like the idea that I wasn't wearing any socks. So, I wear socks when I come in here. I don't wear them normally" (P01).

The burdens of offloading devices were experienced as both physical and psychological consequences, with some participants complaining of physical exhaustion from wearing a removable cast walker every day. Others reported how they were missing out on meaningful activities due to the equipment.

"You walk around for a long time but it's like walking with a concrete brick" (P03).

Social roles were negatively impacted by commitments to ulcer management procedures. Some participants experienced difficulties with caring for grandchildren and attending school drop-off whilst using a removeable cast walker for their ulcer. Difficulties taking the cast walker on and off to drive limited individual freedom to meet friends or attend a workplace.

"It's frustrating not being able to just get in the car and go where I feel like going, makes a difference and friends have to come and see me rather than me going to see them" (P09).

Keeping ulcer dressings dry and clean was also difficult for many participants and prevented participation in outdoor leisure activities including going to the beach, riding a bike or going on walks.

"I can't go to the water. I haven't been in the pool for two years" (P09).

These challenges experienced by participants with ulcer offloading equipment and dressings forced some into a more sedentary lifestyle. A few participants reported positive experiences when podiatrists had demonstrated innovation to adapt the prescribed equipment so their lifestyle burden was reduced.

"Last time she put in extra blocks in the orthotics and now she's put a carbon plate in, she's trying her best" (P07).

\section{Theme 4. It's a long journey}


Podiatric ulcer management was experienced by all participants as a long and interrupted journey. Six participants struggled with acceptance of their ulcer and implementing offloading equipment into their lifestyle. Several participants had almost healed their ulcer completely before experiencing ulcer infection and continued treatment.

"And then it (ulcer) breaks down again. It's a never-ending cycle" (P10).

"It's one step forward and two steps back" (P05).

Participants recognised that despite compliance to regular podiatric ulcer treatment, the healing process for a DFU could not be rushed.

"It's going to take a long time. It's just something that you can't rush. If you rush it, you'll get to a certain point and just break down again. Then you're back to square one" (P10).

Participants were grateful for emotional support and medical attention they received from podiatrists and other health professionals during this long journey.

"There were vascular surgeons, three or four nurses, I felt like a real king" (P07).

However, the journey of podiatric ulcer treatment included unpleasant experiences for individuals. Ulcer infection was a significant setback for several participants which created doubt about timely ulcer resolution. Return to work presented setbacks to ulcer treatment for one participant as the physical requirements of work tasks and the workplace environment were not appropriate for ulcer healing. The interrupted journey of podiatric ulcer management had significant impacts on the wellbeing of participants.

"Your legs are your pillars. So, having any wounds affect your mobility or your way of life is life changing" (P08).

Ulcer infections were commonly experienced and prolonged the treatment journey. Participants described increased responsibilities on a partner to support them throughout their podiatric ulcer treatment. Guilt and frustration were experienced by these participants as their partners were required to increase household tasks and transport them to and from their podiatry appointments. Two participants experienced difficulty maintaining full time employment due to time commitments attending frequent podiatry appointments and use of a removeable cast walker. Consequently, uncertainty towards future job prospects was expressed.

The ulcer was a co-morbidity to other health problems for most participants. Other medical conditions were of greater priority than the ulcer, which was less of a concern for some participants.

"When you've had your chest cut open, this is nothing. It's a fairly minor inconvenience" (P04). 
However, many still experienced significant stress managing podiatric ulcer treatment alongside their comorbidities.

"Having a medical condition on top is like having the weight of the world on your shoulders" (P08).

As a result of countless setbacks whilst receiving podiatric treatment for their ulcer, some participants demonstrated a pessimistic outlook towards timely ulcer resolution and their return to a regular lifestyle.

\section{Discussion}

This study aimed to investigate how people with DFUs experience receiving podiatric ulcer treatment. A person's ability to trust their podiatrist was a key facilitator for a positive treatment experience. Trust was achieved when podiatrists demonstrated professional expertise in communication, ulcer debridement, and dressings. This is consistent with reported experiences of receiving treatment for rheumatoid arthritis-related foot ulcers (34).

The results of this study make a unique contribution to the literature by highlighting the lived experience differences in podiatric care between hospitals and community clinics. Participants conveyed more positive experiences and were more inclined to trust podiatrists in the hospital as they were perceived to demonstrate better hygiene practices and innovation in personalising treatment plans. This finding builds on previous research which has identified trust for health professionals correlates with higher patient satisfaction (35).

Consistency in podiatrist and treatment is another factor that helped people to trust the quality of their podiatry care. A person's familiarity with their health professional has been shown to help establish therapeutic patient-professional relationships in previous studies (34). This emphasises the need for podiatrists to offer consistency of clinician to a person with a DFU. If change of podiatrist is indicated, an explanation describing the rationale for this change in relation to the person's individual ulcer circumstance and confirming the expertise of the new podiatrist could reduce patient distrust.

The study's findings suggest that podiatrists need to provide individualised information to people with DFUs for the information to be useful to the person. This is supported by Calnan and Rowe (36) who highlighted the importance of tailoring health advice to meet patient needs and establish trust. Participants' desires for meaningful information in this study is consistent with the expectations of information in studies of experiences of other foot-related conditions. A study of individuals with plantar heel pain indicated they wanted clear explanations about their condition's aetiology and treatment (26). However, this desire for information was not coherent amongst all participants in this study with some expressing indifference to information provided by podiatrists. This highlights that personalising information includes tailoring it to both the individual's expectations and their health literacy to facilitate positive outcomes. The study findings support open communication facilitated by a trusted relationship can enable podiatrists to gain an insight into an individual's challenges to tailor information and interventions to their needs. 
Podiatrists providing meaningful information to their patients with DFUs may be beneficial in encouraging treatment compliance for successful ulcer outcomes. The Transtheoretical Model of Change describes the stages of change in health behaviour, which can be experienced in different sequences (Figure 3) (37). At the pre-contemplation or contemplation stages, awareness raising through tailored education about a person's ulcer management can support positive behavioural change (37).

Personalised information from a podiatrist can assist individuals with preparation and action such as keeping ulcer dressings clean and compliance with offloading equipment. Podiatrists should aim to develop individualised treatment plans to suit a person's stage of change and optimise treatment outcomes (38). These results must be considered with the caveat that the theme of "personalised information" was comprised of the smallest data set of the study themes. The variation in patient expectations for information from their podiatrist that was highlighted in this study warrants further investigation into how podiatrists can best personalise ulcer treatment to the individual to enhance experiences of ulcer treatment and encourage compliance to ulcer management procedures.

This study proposes that compliance can also be supported by podiatrists being innovative in meeting the person's needs in ulcer management. Prescribed equipment including removable cast walkers and dressings were a significant burden in daily activities for many participants. Previous studies have described the lifestyle limitations presented by ulcer offloading devices (39). Ulcer equipment was a burden to family and carer roles for some participants. This is supported by Kinmond et al. (40) who reported restrictions in activity participation and relationships as a result of daily use of ulcer equipment. These lifestyle limitations can affect an individual's quality of life and psychological wellbeing living with a DFU (40). This study found that even when participants had trust in their podiatrist and understood instructions about ulcer care, the limitations of the equipment could make it too difficult for them to comply. Willingness of the podiatrist to adapt equipment or trial different equipment to reduce limitations in meaningful activities was perceived positively and improved the person's capacity to comply with podiatric treatment.

Setbacks in ulcer recovery during podiatry treatment were experienced by participants as negative impacts on employment, personal independence, and return to meaningful activities. This is consistent with current literature describing lifestyle burden from ulcer management including a sense of guilt due to an increased dependence on others for daily needs $(2,9)$. Our results indicated that the use of dressings and offloading devices significantly contribute to lifestyle burden. Moreover, commitments to podiatric ulcer treatment between appointments limited opportunities for full time employment as participants felt pressured to keep their ulcer clean, dry, and protected to promote timely healing and felt unable to manage this in a workplace. Although no participants expressed a financial burden due to this occupational limitation, which has been previously described in the literature $(2,23)$, this may have been because most of the sample were retired.

Living with a chronic illness such as diabetes mellitus can introduce substantial psychological impacts as people with the chronic illness can struggle with compliance to prescribed treatment (41). Consistent with previous research involving people with diabetes mellitus, many participants in this study struggled 
with acceptance of their ulcer and the timeline and burden of its associated podiatric treatment (41). Participants demonstrated a pessimistic outlook for their foot health which has also been identified in other studies (34). To support the psychological health of people receiving treatment for a DFU, it is important for health professionals to empathise with the person and attempt to understand the lifestyle impacts of ulcer treatment in order to tailor treatment strategies to minimise burden and promote positive patient experiences.

\section{Limitations and further research}

Limitations of this study must be considered before implementing the findings into clinical practice. Information about the participant's ulcer including infection, duration of ulcer and podiatric treatment was self-reported, no medical records were accessed, and the researchers did not assess the participant's ulcer. Semi-structured interviews provide participants with one opportunity to report their lived experiences and therefore, they may not provide a full report of their experience if they do not recall the details at the time of the interview.

A sampling bias may have influenced the lived experiences described in this study. All participants were receiving public sector healthcare and many participants were referred from other hospitals within the same local health network. Therefore, these experiences may not be reflective of people receiving private healthcare only or people living in other locations. Future research to understand the lived experience of podiatry care for people with DFUs could compare experiences between public and private healthcare or investigate experiences of podiatric ulcer treatment across metropolitan and rural South Australia.

All participants in this study were male as the majority of people receiving DFU treatment in that particular podiatry department were male. People who associate with other genders may have other values which influence their experience of podiatric ulcer management including familial roles and expectations with use of ulcer equipment. Additionally, all participants were not employed at the time of the study. Further research can improve our understanding of the impacts of podiatric ulcer treatment on occupation.

\section{Conclusion}

This study found that podiatric ulcer treatment is a lengthy and onerous journey for many people with DFUs. The podiatric management of ulcers with offloading equipment and dressings was shown to be a significant contributor to treatment burden and can have negative impacts on a person's independence in social and daily activities. People with DFUs felt confident in their ulcer treatment when they trusted their podiatrist. A trusting relationship was supported by consistency in the podiatrist who provided the ulcer treatment and demonstration of high organisational and hygiene standards. A podiatrist's willingness to personalise information to address an individual's concerns and be innovative in adaptation of ulcer equipment to minimise the burden on a person's roles and responsibilities can improve a person's experiences with podiatric treatment for a DFU. Consideration by podiatrists of a person's physical, 
occupational, and psychosocial lifestyle factors which impact their experience with podiatric ulcer treatment will support development of a personalised and achievable treatment plan.

\section{Declarations}

\section{Ethics approval and consent to participate}

Ethics approval was obtained from the University of South Australia (project number 203393) and Central Adelaide Local Health Network (reference number 13567) human research ethics committee. All participants provided written informed consent for their participation in this study.

\section{Consent for publication}

Consent forms signed by the participants stated that the results of the study would be used for publication.

\section{Availability of data and materials}

Transcripts will not be made available to maintain confidentiality of the participants.

\section{Competing interests}

The authors declare no competing interests.

\section{Funding}

No funding involved in this study.

\section{Authors' contributions}

EO: Formal analysis, investigation, data curation, writing- original draft preparation, writing- review \& editing, visualisation. RC: Conceptualisation, methodology, writing- review \& editing, supervision. KG: Conceptualisation, methodology, writing- review \& editing, supervision. CF: Conceptualisation, methodology, writing- review \& editing, supervision.

\section{Acknowledgements}

The authors would like to acknowledge all the participants who volunteered to be involved in this study. We would also like to thank the podiatrists at The Queen Elizabeth Hospital, in particular Cathy Loughry, Judith Sharp, Madeline Primavera, Hannah Snelling, and Joe Beilby for their assistance in identifying and recruiting participants for this study.

\section{References}


1. Grażyna R, Grażyna N, Renata S, Kazimiera Z, Jana N. Problems related to functioning and quality of life of patients with diabetic foot ulcers. Studia Medyczne. 2013;29(4):338-42.

2. Vileikyte LO. Diabetic foot ulcers: A quality of life issue. Diabetes Metab Res Rev. 2001;17(4):246-9.

3. Polikandrioti M, Vasilopoulos G, Koutelekos I, Panoutsopoulos G, Gerogianni G, Babatsikou F, et al. Quality of life in diabetic foot ulcer: Associated factors and the impact of anxiety/depression and adherence to self-care. Int J Low Extrem Wounds. 2020;19(2):165-79.

4. Reiber GE, Vileikyte LO, Boyko ED, Del Aguila M, Smith DG, Lavery LA, et al. Causal pathways for incident lower-extremity ulcers in patients with diabetes from two settings. Diabetes Care. 1999;22(1):157-62.

5. Peat G, Rodriguez A, Smith J. Interpretive phenomenological analysis applied to healthcare research. Evid Based Nurs. 2019;22(1):7-9.

6. Wu S, Jensen J, Weber A, Robinson D, Armstrong D. Use of pressure offloading devices in diabetic foot ulcers: Do we practice what we preach? Diabetes Care. 2008;31(11):2118-9.

7. St Clair VW. Doing (interpretive) phenomenology. In: Nayar S, Stanley M, editors. Qualitative research methodologies for occupational science and therapy. London: Routledge; 2014. p. 53-69.

8. Diabetic Foot Australia: The estimated burden of diabetes-related foot disease in Australia in 2017. https://diabeticfootaustralia.org/wp-content/uploads/DFA-Guides-You-Through-The-estimatedburden-of-diabetes-related-foot-disease-in-Australia-in-2017-Final-PDF-241017.pdf (2017). Accessed 23 Mar 2020.

9. Meriç M, Ergün G, Meriç C, Demirci I, Azal Ö. It is not diabetic foot: It is my foot. J Wound Care. 2019;28(1):30-7.

10. Moura Neto A, Zantut-Wittmann D, Fernandes T, Nery M, Parisi M. Risk factors for ulceration and amputation in diabetic foot: Study in a cohort of 496 patients. Endocrine. 2013;44(1):119-24.

11. Armstrong D, Boulton A, Bus S. Diabetic foot ulcers and their recurrence. N Engl J Med. 2017;376(24):2367-75.

12. Alavi A, Sibbald R, Mayer D, Goodman L, Botros M, Armstrong D, et al. Diabetic foot ulcers: Part I. Pathophysiology and prevention. J Am Acad Dermatol. 2014;70(1):e1-18.

13. Jupiter D, Thorud J, Buckley C, Shibuya N. The impact of foot ulceration and amputation on mortality in diabetic patients. I: From ulceration to death, a systematic review. Int Wound J. 2016;13(5):892903.

14. Benkel I, Arnby M, Molander U. Living with a chronic disease: A quantitative study of the views of patients with a chronic disease on the change in their life situation. SAGE Open Med. 2020;8:1-7.

15. Trikkalinou A, Papazafiropoulou A, Melidonis A. Type 2 diabetes and quality of life. World J Diabetes. 2017;8(4):120-9.

16. Dewi F, Hinchliffe R. Foot complications in patients with diabetes. Surgery 2020;38(2):108-13.

17. Van Netten J, Seng L, Lazzarini P, Warnock J, Ploderer B. Reasons for (non-) adherence to self-care in people with a diabetic foot ulcer. Wound Repair Regen. 2019;27(5):530-9. 
18. Beach M, Keruly J, Moore R. Is the quality of the patient-provider relationship associated with better adherence and health outcomes for patients with HIV? J Gen Intern Med. 2006;21(6):661-5.

19. Kaplan S, Greenfield S, Ware J. Assessing the effects of physician-patient interactions on the outcomes of chronic disease. Med Care. 1989;27(3):S110-S27.

20. Nemcová J, Hlinková E, Farský I, Žiaková K, Jarošová D, Zeleníková R, et al. Quality of life in patients with diabetic foot ulcer in Visegrad countries. J Clin Nurs. 2017;26(9-10):1245-56.

21. Vileikyte LO. Psychosocial and behavioral aspects of diabetic foot lesions. Curr Diab Rep. 2008;8(2):119-25.

22. McPherson M, Binning J. Chronic foot ulcers associated with diabetes: Patients' views. Diabet foot. 2002;5(4):198.

23. Brod M. Quality of life issues in patients with diabetes and lower extremity ulcers: Patients and care givers. Qual Life Res. 1998;7(4):365-72.

24. Foster D, Lauver L. When a diabetic foot ulcer results in amputation: A qualitative study of the lived experience of 15 patients. Ostomy Wound Manag. 2014;60(11):16-22.

25. Campbell R, Morriss-Roberts C, Durrant B, Cahill S. "I need somebody who knows about feet" a qualitative study investigating the lived experiences of conservative treatment for patients with posterior tibial tendon dysfunction. JFAR. 2019;12(1):51.

26. Cotchett M, Rathleff M, Dilnot M, Landorf K, Morrissey D, Barton C. Lived experience and attitudes of people with plantar heel pain: A qualitative exploration. JFAR. 2020;13(1):12.

27. Tong A, Sainsbury P, Craig J. Consolidated criteria for reporting qualitative research (COREQ): A 32item checklist for interviews and focus groups. Int J Qual Health Care. 2007;19(6):349-57.

28. Matua G, Van Der Wal D. Differentiating between descriptive and interpretive phenomenological research approaches. Nurs Res. 2015;22(6):22-7.

29. Morse J. Critical analysis of strategies for determining rigor in qualitative inquiry. Qual Health Res. 2015;25(9):1212-22.

30. Murray C, Wilde D. Thinking about, doing and writing up research using interpretative phenomenological analysis. In: Walshe C, Brearley S, editors. Handbook of theory and methods in applied health research. Gloucestershire: Edward Elgar Publishing; 2020. p. 140-66.

31. Guba E, Lincoln Y. Fourth generation evaluation. 1st ed. California: Sage Publications; 1989.

32. Korstjens I, Moser A. Series: Practical guidance to qualitative research. Part 4: Trustworthiness and publishing. Eur J Gen Pract. 2018;24(1):120-4.

33. Saunders B, Sim J, Kingstone T, Baker S, Waterfield J, Bartlam B, et al. Saturation in qualitative research: Exploring its conceptualization and operationalization. Qual Quant. 2018;52(4):1893-907.

34. Firth J, Nelson A, Briggs M, Gorecki C. Experiences of healthcare provision for foot ulceration occurring in people with rheumatoid arthritis. Musculoskeletal Care. 2013;11(3):159-67.

35. Birkhauer J, Gaab J, Kossowsky J, Hasler S, Krummenacher P, Werner C, et al. Trust in the health care professional and health outcome: A meta-analysis. PLoS One. 2017;12(2):e0170988. 
36. Calnan M, Rowe R. Trust matters in health care. 1st ed. Berkshire: McGraw-Hill Education; 2008.

37. Prochaska J, Velicer W. The transtheoretical model of health behavior change. Am J Health Promot. 1997;12(1):38-48.

38. Zimmerman GL, Olsen C, Bosworth M. A 'stages of change' approach to helping patients change behavior. Am Fam Physician. 2000;61(5):1409-16.

39. Coffey L, Mahon C, Gallagher P. Perceptions and experiences of diabetic foot ulceration and foot care in people with diabetes: A qualitative meta-synthesis. Int Wound J. 2019;16(1):183-210.

40. Kinmond K, McGee P, Gough S, Ashford R. 'Loss of self': A psychosocial study of the quality of life of adults with diabetic foot ulceration. J Tissue Viability. 2003;13(1):6-16.

41. Silva J, Souza E, Böschemeier A, Costa C, Bezerra H, Feitosa E. Diagnosis of diabetes mellitus and living with a chronic condition: Participatory study. BMC Public Health. 2018;18(1):699.

\section{Appendix}

Appendices 1, 2 and 4 are not available with this version.

\section{Figures}




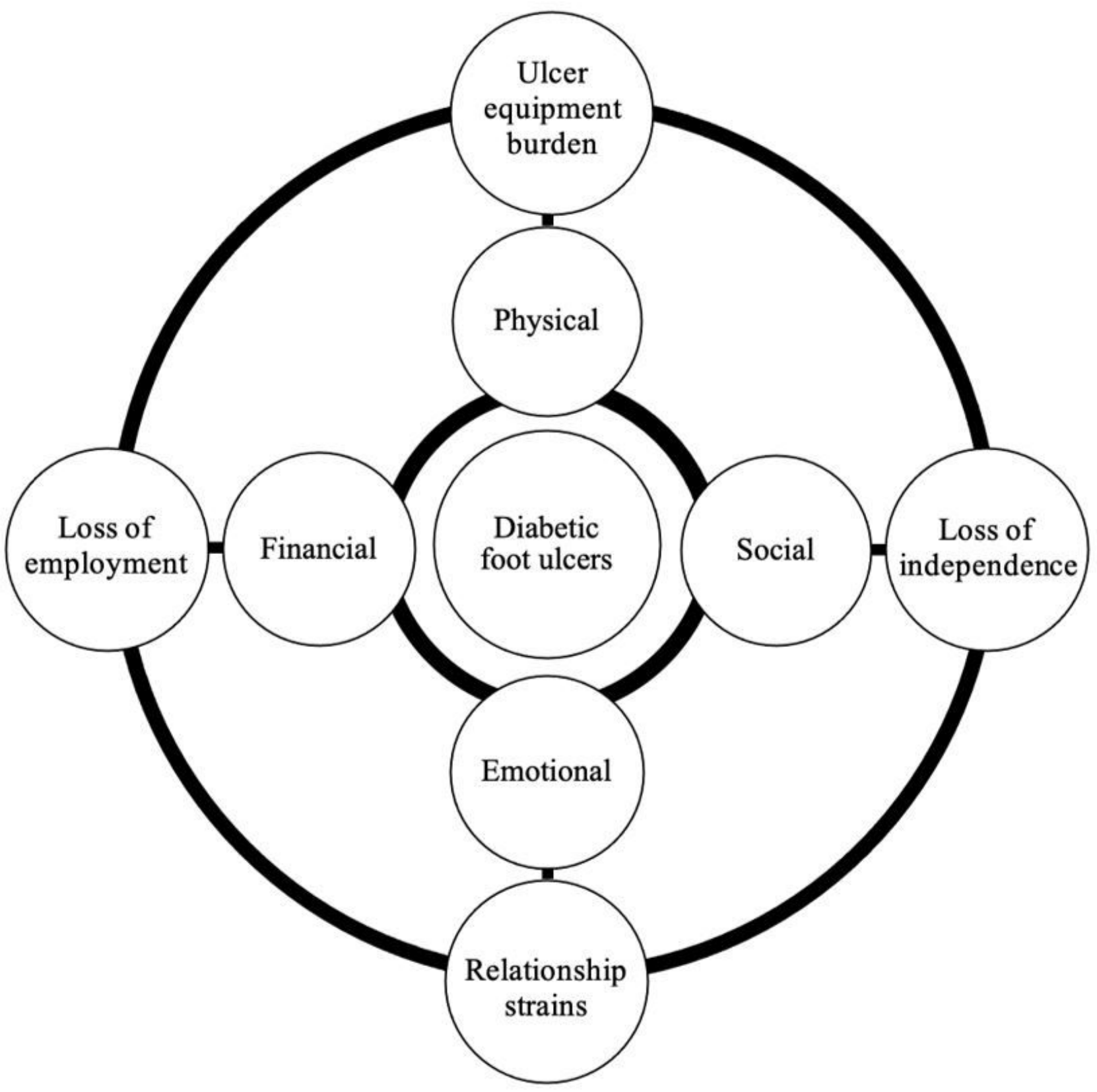

Figure 1

Multi-faceted impact of diabetic foot ulcers on patients $(1,2)$ 
1. Trusting the podiatrists with the right expertise
2. Personalised information

How do people living with diabetic foot ulcers experience receiving podiatric management?

3. Happy with the service, not the equipment

Figure 2

Four themes 


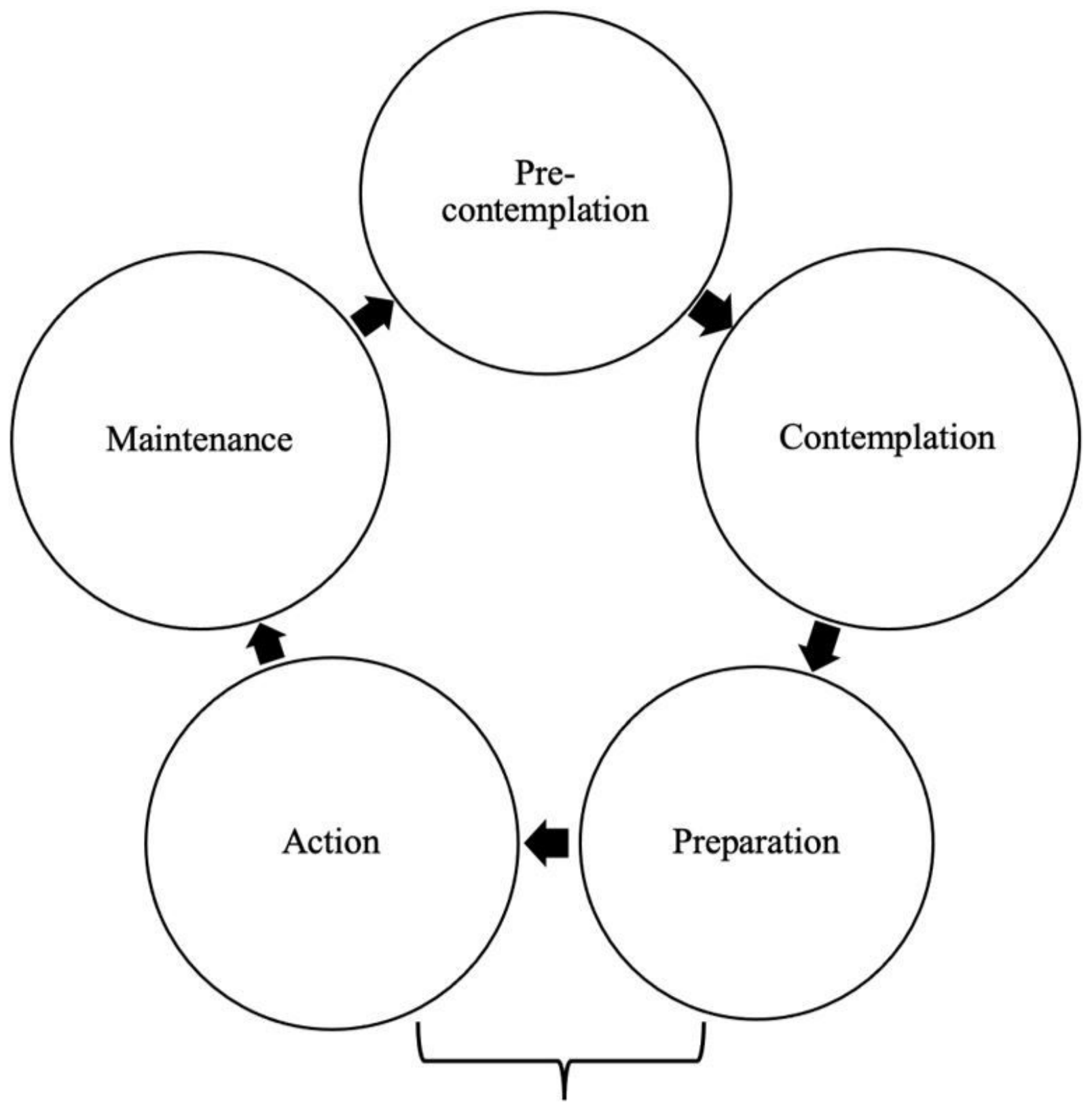

Podiatrists providing personalised information and care to patients to encourage treatment compliance

Figure 3

Transtheoretical Model of Change (37)

\section{Supplementary Files}

This is a list of supplementary files associated with this preprint. Click to download.

- Additionalparticipantdemographicdata.docx 
- Interviewquestions.docx

Page 23/23 